\title{
Gentamicin ototoxicity: a 23-year selected case series of 103 patients
}

\section{Rebekah M Ahmed \\ MB BS, FRACP. \\ Neurology Fellow \\ Imelda P Hannigan \\ RN, Neuro-otology Nurse \\ Hamish G MacDougall \\ Vestibular Scientist}

Raymond C Chan

MB BS, FRACP, FRCPA Infectious Disease Physician

G Michael Halmagyi MD, FRACP
Neurologist

1 Department of Neurology, Royal Prince Alfred Hospital, Sydney, NSW.

2 Vestibular Research

Laboratory, School of

Psychology, University of

Sydney, Sydney, NSW.

gmh@icn.usyd.edu.au

MJA 2012; 196: 701-704 doi:10.5694/mjall.10850

Editorial p 665

Online first 03/04/12 entamicin is an important bactericidal antibiotic with two serious potential adverse effects: nephrotoxicity and ototoxicity. Clinicians are well aware that rising serum creatinine levels in patients treated with gentamicin could indicate nephrotoxicity. However, many do not know that, contrary to textbooks and antibiotic guidelines, gentamicin ototoxicity causes impairment of vestibular, not auditory, function. ${ }^{1,2}$ Vestibulotoxicity is frequently overlooked in patients having gentamicin, ${ }^{3-7}$ so that severe, irreversible, bilateral vestibular loss can occur, causing permanent imbalance, which is particularly debilitating in elderly people.

We reviewed patients presenting to a balance disorders clinic between 1988 and 2010 with severe, selective, bilateral vestibular loss, who had been treated in hospital with gentamicin between 1975 and 2010.

\section{Methods}

\section{Patients}

Over the 23-year period, 552 patients were diagnosed with severe, symmetrical, selective (ie, normal hearing for age), bilateral vestibular loss. Of these, 263 patients had gentamicin vestibulotoxicity (GVT). In the remainder, the vestibular loss was caused by cisplatinum ototoxicity (9), meningitis (6), hereditary factors (29), or bilateral sequential vestibular neuritis (61); in 184 the condition was idiopathic. ${ }^{8}$

Patients with GVT who had severe bilateral vestibular loss after having gentamicin in hospital were interviewed and examined, and hospital records for the "gentamicin admission" were obtained. Our study included 103 patients with normal renal function at the start of gentamicin treatment (serum creatinine level, <121 $\mu \mathrm{mol} / \mathrm{L}$ [eGFR, > $59 \mathrm{~mL} /$ $\left.\mathrm{min} / 1.73 \mathrm{~m}^{2}\right]$ ) and for whom the following data were available: (i) total dose and dosage regimen of gentamicin; (ii) renal function before and during treatment; (iii) serum gentamicin level; (iv)

\section{Abstract \\ Objective: To review patients with severe bilateral vestibular loss associated with gentamicin treatment in hospital.}

Design and setting: A retrospective case series of presentations to a balance disorders clinic between 1988 and 2010.

Main outcome measures: Relationship between vestibulotoxicity and gentamicin dose or dosing profile; indications for prescribing gentamicin.

Results: 103 patients (age, 18-84 years; mean, 64 years) presented with imbalance, oscillopsia or both, but none had vertigo. Only three noted some hearing impairment after having gentamicin, but audiometric thresholds for all patients were consistent with their age. In all patients, the following tests gave positive results: a bilateral clinical head-impulse test, a vertical head-shaking test for vertical oscillopsia, and a foam Romberg test. In 21 patients, imbalance occurred during gentamicin treatment (ignored or dismissed by prescribers in 20) and in 66 after treatment; the remaining 16 could not recall when symptoms were first noticed, except that it was after gentamicin treatment in hospital. Total gentamicin dose range was $2-318 \mathrm{mg} / \mathrm{kg}$ (mean, $52 \mathrm{mg} / \mathrm{kg}$ ), daily dose range was $1.5-5.6 \mathrm{mg} / \mathrm{kg}$ (mean, $3.5 \mathrm{mg} / \mathrm{kg}$ ), and duration was $1-80$ days (mean, 17 days). Six patients had only a single dose; 26 had five or fewer doses. Serum gentamicin levels, measured in 82 patients, were in the recommended range in 59. Time to diagnosis ranged from 4 days to 15 years. Nephrotoxicity developed in 43 patients. Gentamicin dosage complied with contemporary or current Australian antibiotic guidelines in under half the patients.

Conclusions: Gentamicin ototoxicity is vestibular, not cochlear, producing permanent loss of balance, but not of hearing. Gentamicin can be vestibulotoxic in any dose, in any regimen, at any serum level.

indications (clinical and microbiological) for using gentamicin; (v) when imbalance was first noted by the patient; and (vi) when bilateral vestibular loss was first clinically recognised.

Ethics approval was not required for this retrospective review.

\section{Diagnosis}

\section{Vestibular loss}

Bilateral vestibular loss was diagnosed if all the following gave positive results: (i) bi-directional, horizontal and vertical head impulse test (Videos 1-4); ${ }^{9}$ (ii) vertical oscillopsia with loss of at least three lines of visual acuity on a Snellen chart during vertical head shaking (Video 5) ${ }^{10}$ and (iii) negative results of a Romberg test on a firm surface and positive results on a foam surface (Video 6) (Videos 1-6; available at mja.com.au). ${ }^{11}$

\section{Vestibular testing}

To confirm the diagnosis and quantify vestibular loss, 95 patients with GVT (the remaining eight were too frail to test) had either caloric or rotational vestibular testing of lateral semicircular canal function, or both (Box 1; available at mja.com.au). ${ }^{12}$

\section{Auditory testing}

Air-conduction, pure-tone threshold, clinical audiometric graphs (0.25$8 \mathrm{kHz})$, from hearing measurement in each ear when GVT was diagnosed, were available in 73 of the 103 patients. Frequencies above $8 \mathrm{kHz}$ were not tested. In patients with air-conduction thresholds above normal for age and in those with middle-ear disease, bone-conduction thresholds were also measured. Audiologists' descriptive reports of the audiometric results were available for the remaining 30 patients.

\section{Indications for gentamicin}

We reviewed patients' clinical diagnoses to determine (i) whether gentamicin therapy complied with contemporary (at the time of admission to hospital) or with current (2010) Australian antibiotic guidelines; ${ }^{13}$ and (ii) whether the treatment was empirical, but appropriate, or based on results of cultures. 


\section{Results}

The 103 patients who fulfilled our criteria comprised 47 men, 56 women; mean age, 64 years (range, 18-84 years). Forty-eight presented with both imbalance and oscillopsia, 39 with imbalance only, and four with oscillopsia only; and in 12 patients we were unable to determine their main presenting symptom. In all patients, the following tests gave positive results: a bilateral clinical head impulse test, ${ }^{9}$ a vertical head-shaking test for vertical oscillopsia, ${ }^{10}$ and a foam Romberg test (Videos 1-6; available at mja.com.au). ${ }^{11}$ Thirty-eight patients had recurrent falls or required a walking aid, and 44 required vestibular rehabilitation. ${ }^{14}$

Twenty-one patients first noted symptoms in hospital during gentamicin treatment (gentamicin treatment was stopped in only one patient); 29 patients had completed treatment but were still in hospital; 37 experienced symptoms after discharge; and 16 could not remember when they first noticed symptoms, except that it was after gentamicin treatment in hospital.

\section{Time to diagnosis}

The delay to diagnosis of bilateral vestibular loss ranged from 4 days (the only patient in whom GVT was diagnosed during treatment) to 15 years. GVT was diagnosed less than 12 months after treatment in 69 patients, and more than 12 months after treatment in 34 patients.

\section{Absence of cochleotoxicity}

Only three of the 103 patients with GVT complained of hearing impairment after gentamicin treatment, in each case while still in hospital. No patient had audiometry at the time gentamicin was given, but all had audiometry later as part of the assessment of vestibular loss. For the 73 patients whose audiograms were retrieved, hearing loss at each frequency, averaged for each age group

4 Trough or peak levels of serum gentamicin $(\mu \mathrm{g} / \mathrm{mL})(n=82)$

Trough, mean

(range)*

Peak, mean (range) ${ }^{\dagger}$

\begin{tabular}{llll}
\hline All patients $^{\ddagger}$ & $2.4(0-12.9)$ & $(n=51)$ & $5.3(0.3-19.0)(n=53)$ \\
No nephrotoxicity & $1.8(0-12.9)$ & $(n=26)$ & $4.6(0.3-19.0)(n=28)$ \\
Nephrotoxicity & $3.4(0.6-6.6)$ & $(n=25)$ & $5.9(1.4-13.5) \quad(n=25)$
\end{tabular}

* Reference interval, $0.5-2.0 \mu \mathrm{g} / \mathrm{mL}$. † Reference interval, $4.0-10.0 \mu \mathrm{g} / \mathrm{mL}$.

$\ddagger \ln 21$ patients there was no record of either a trough or a peak serum level. and both ears, was not different from accepted age-group means (Box 2; available at mja.com.au). ${ }^{15,16}$ Audiometry in the three patients who complained of hearing loss also showed thresholds consistent with age. The pure-tone thresholds in the 30 descriptive reports were reported as normal or showing only high-frequency hearing loss, consistent with age and noise exposure. We were unable to retrieve audiometry data from before gentamicin treatment for any of the patients.

\section{Nephrotoxicity}

During gentamicin treatment 43 of 103 patients developed nephrotoxicity, defined as a serum creatinine level $>120 \mu \mathrm{mol} / \mathrm{L}$ (range, 121-841 $\mu \mathrm{mol} / \mathrm{L}$ ) and eGFR $<60 \mathrm{~mL} / \mathrm{min} / 1.73 \mathrm{~m}^{2}$ (range, $59-<6 \mathrm{~mL} / \mathrm{min} / 1.73 \mathrm{~m}^{2}$ ) on two sequential daily measurements.

\section{Dosage and administration}

The gentamicin dose ranged from 160 to $320 \mathrm{mg} /$ day, equivalent to 1.5 to $5.6 \mathrm{mg} / \mathrm{kg} /$ day (mean, $3.5 \mathrm{mg} / \mathrm{kg} /$ day). The total gentamicin dose ranged from 160 to $16520 \mathrm{mg}$, equivalent to 2$318 \mathrm{mg} / \mathrm{kg}$ (mean, $3639 \mathrm{mg}$ or $52 \mathrm{mg} /$ $\mathrm{kg})$. The mean total dose in the 43 patients who developed nephrotoxicity was $4363 \mathrm{mg}$, in contrast to $3240 \mathrm{mg}$ in the 60 who did not. The gentamicin doses our patients with GVT received in hospital, between 1975 and 2010, indicate that there was no change in the total dose or daily dose of gentamicin given in this period. Box 3 (available at mja.com.au) shows total dose $(\mathrm{mg} / \mathrm{kg}$ ) versus daily dose $(\mathrm{mg} /$ $\mathrm{kg} /$ day) for each patient.

Fifty-five patients received only one daily dose of gentamicin; 14 received three doses per day, nine received two to three doses per day, 11 received two doses per day, one received a combination of one and three doses per day, and two received four doses per day. For 11 patients, we were unable to obtain reliable data on doses per day given. The total number of doses over the treatment period ranged from one to 129 (mean, 30). Twenty-six patients received five or fewer doses of gentamicin and six patients received a single dose only.

Duration of treatment ranged from 1 day (the six patients who had only a single dose) to 80 days (mean, 17 days).
It was not possible from the treatment chart information to determine the exact method of intravenous administration used: slow infusion (recommended) or bolus injection.

\section{Serum gentamicin levels}

Serum gentamicin levels (trough or peak or both) were retrieved in 82 patients; in 23, a trough or a peak level was above the recommended range. Peak levels were higher in those who developed nephrotoxicity than in those who did not (Box 4).

\section{Indications and results of culture}

Adherence to contemporary or current antibiotic guidelines for gentamicin use $^{13}$ is shown by patient diagnosis in Box 5 and by clinician subspecialty in Box 6.

Culture results were available in 73 of 103 patients; 44 showed no growth. An organism was isolated in 29 cultures, sensitive to gentamicin in 11 ; in another 11 gentamicin was not indicated for treatment of the organism isolated (eg, methicillin-sensitive Staphylococcus aureus); and in seven gentamicin sensitivity was not tested or reported.

\section{Discussion}

\section{Loss of balance, not of hearing}

All these patients developed symptoms and signs of bilateral vestibular impairment after treatment with gentamicin, but only three noted any hearing impairment. Audiometric thresholds in the all patients were not significantly different from age-matched thresholds. ${ }^{15,16}$ Unless pre-gentamicin audiometry is available, high-frequency (4$8 \mathrm{kHz}$ ) hearing loss, especially in elderly men, cannot be assumed to be due to gentamicin; noise and ageing are much more common causes of hearing loss. ${ }^{1}$ Measuring hearing before and after gentamicin treatment ${ }^{17,18}$ shows only slight (about $15 \mathrm{~dB}$ ) high-frequency $(4-8 \mathrm{kHz})$, asymptomatic hearing loss. This suggests that any hearing loss from gentamicin ototoxicity would not be noticed by patients with normal hearing, ${ }^{19,20}$ and certainly not by those with pre-existing, high-frequency hearing loss. By contrast, symptoms of vestibular loss are obvious to patients, as are the clinical signs to the aware clinician. 


\section{Delayed recognition}

All patients noted imbalance either during hospital admission or immediately after discharge. We were able to confirm that they had received gentamicin and had no other potential cause for bilateral vestibular loss. Even though it has been known since $1952^{21}$ that certain aminoglycosides, including streptomycin and gentamicin, can cause severe, selective loss of vestibular function, ${ }^{1-8}$ prescribers rarely recognise GVT. ${ }^{3,4}$

Because vestibular loss is, at least initially, bilateral and symmetrical, patients will develop imbalance and oscillopsia, not vertigo, the hallmark of acute, unilateral vestibular loss. Bed-bound patients will be unaware of their imbalance until they walk again. Unless clinicians know how to test for bilateral vestibular loss, gentamicin treatment will not be stopped and vestibulotoxicity will most likely be aggravated. Bilateral vestibular loss can now be objectively confirmed at the bedside using video-vestibulometry (Box 7; Video 3, Video 4; available at mja.com.au), ${ }^{22}$ dynamic visual acuity $^{10}$ and the foam Romberg test (in ambulant patients) (Video 6; available at mja.com.au). ${ }^{11}$

Even when patients report that imbalance developed after hospital admission, gentamicin is rarely considered as the possible cause. With delayed diagnosis, it is difficult without a subpoena ${ }^{23}$ to obtain hospital drug treatment charts to confirm that gentamicin was given.

\section{Dosage and administration}

There has not been any change in the overall gentamicin dose given to patients who develop GVT since $1994 .^{3}$ We found that regardless of total dose, daily dose, number of doses per day or duration of treatment, patients can still develop GVT. We also confirmed that GVT can occur even when serum trough or peak levels are at or below the recommended range. ${ }^{3,4}$

In all but one of our patients, GVT was not diagnosed during treatment, and six patients had only a single gentamicin dose. Thus, 96 of our patients would have continued being given gentamicin after developing vestibulotoxicity (many were bed- bound), so that the total dose given to these 96 patients must have exceeded the toxic dose.

A study of 33 patients with GVT also found no relationship between daily dose, total dose or serum levels and GVT, and 32 of their patients were diagnosed after hospital discharge. ${ }^{4}$

\section{Link with nephrotoxicity}

The mean total dose and serum levels of gentamicin in the 43 patients with GVT who developed nephrotoxicity ${ }^{24,25}$ were substantially higher than in the 60 who did not. Patients who develop vestibulotoxicity seem more likely to develop nephrotoxicity $(42 \%)$ than patients in general receiving gentamicin $(5 \%-17 \%),{ }^{26}$ suggesting a common mechanism or predisposing factor.

\section{Guideline indications}

The current (2010) Australian antibiotic guidelines advise that use of gentamicin for empirical treatment should be limited to 48 hours, pending the results of microbiological investigations. Directed therapy is indicated only for infections in which there is resistance to other, safer, antimicrobials; for combination therapy in serious Pseudomonas aeruginosa and Brucella infections; and as synergistic treatment for streptococcal or enterococcal endocarditis. It should be considered for prophylactic use only in patients at specific risk of developing endocarditis from genitourinary or gastrointestional procedures. ${ }^{13}$

Gentamicin was given according to current antibiotic guidelines ${ }^{13}$ in $47 \%$ of our 103 patients; $52 \%$ were given empirical gentamicin for longer than the recommended 48 hours; and in only $46 \%$ of patients was gentamicin given in accordance with contemporary antibiotic guidelines. Few medical and surgical specialists prescribed gentamicin based on culture results, and in some cases continued it even when culture showed that it was not indicated.

Previous studies have examined the inappropriate use of aminoglycosides; ${ }^{27,28}$ in one, $10.2 \%$ of antibioticdays were deemed inappropriate. ${ }^{29}$ An education program for junior medical officers improved appropriate prescribing of gentamicin from $52 \%$ to $78 \%{ }^{30}$
5 Diagnoses and compliance with current or contemporary guideline indications for gentamicin; for current indications, number with appropriate duration $(<48 \mathrm{~h})$

\begin{tabular}{|c|c|c|c|}
\hline \multirow[b]{3}{*}{ Diagnosis } & \multicolumn{3}{|c|}{ Number of patients } \\
\hline & \multirow[b]{2}{*}{ Total } & \multicolumn{2}{|c|}{ Guideline indications } \\
\hline & & Current $(<48 \mathrm{~h})$ & Contemporary \\
\hline Endocarditis & 18 & $18(18)$ & 18 \\
\hline Pneumonia & 12 & $1(1)$ & 1 \\
\hline Sepsis & 3 & $3(0)$ & 3 \\
\hline $\begin{array}{l}\text { Urinary tract infection/ } \\
\text { sepsis }\end{array}$ & 9 & $6(3)$ & 6 \\
\hline Febrile neutropenia & 7 & O (na) & 7 \\
\hline Wound infection/ulcer & 10 & $O(n a)$ & 0 \\
\hline Peritonitis & 6 & $6(0)$ & 5 \\
\hline Surgical prophylaxis & 8 & 5* (1) & 5 \\
\hline Biliary sepsis & 9 & $9(0)$ & 2 \\
\hline Pancreatitis & 2 & o (na) & \\
\hline $\begin{array}{l}\text { Septic joint/ } \\
\text { osteomyelitis }\end{array}$ & 15 & $0(0)$ & 0 \\
\hline Thrombophlebitis & 2 & O (na) & 0 \\
\hline $\begin{array}{l}\text { Other (otitis externa, } \\
\text { vascular abscess) }\end{array}$ & 2 & 0 (na) & 0 \\
\hline Total & 103 & $48 / 103(23 / 48)$ & $47 / 103$ \\
\hline
\end{tabular}

na $=$ not applicable as gentamicin not indicated on current guidelines. * In 4 of 5 , gentamicin was listed as second choice.

6 Specialists prescribing gentamicin and compliance with current or contemporary antibiotic guideline indications

\begin{tabular}{lrrr} 
& \multicolumn{3}{c}{ Number of patients } \\
\cline { 2 - 4 } & & \multicolumn{2}{c}{ Guideline indications } \\
\cline { 2 - 4 } Specialty & Total & Current & Contemporary \\
\hline Cardiology; cardiac surgery & 20 & 20 & 20 \\
General surgery & 29 & 17 & 9 \\
\hline General medicine & 15 & 7 & 7 \\
\hline Urological surgery & 3 & 2 & 2 \\
\hline Gynaecological surgery & 1 & 1 & 1 \\
\hline Haematology & 7 & 0 & 7 \\
\hline Rheumatology & 1 & 0 & 0 \\
\hline Geriatric medicine & 2 & 0 & 0 \\
\hline Respiratory medicine & 9 & 1 & 1 \\
\hline Orthopaedic surgery & 16 & 0 & 0 \\
\hline Total & 103 & 48 & 47
\end{tabular}

\section{Study limitations}

As our study did not include a control group, it is not possible, from our data alone, to establish an unequivocal causal link between gentamicin and vestibulotoxicity. However, published clinical $^{1-8}$ and experimental ${ }^{31,32}$ studies remove any doubt.

\section{Minimising vestibulotoxicity}

As there is always a risk of vestibulotoxicity with gentamicin, regardless of dose or serum level, it should be given only as recommended by antibiotic guidelines $^{13}$ and when there is no safer alternative. Clinicians prescribing 
gentamicin should use bedside methods to monitor for vestibulotoxicity, ${ }^{10,22}$ although this is possible only in conscious, cooperative patients. Stopping gentamicin treatment early could prevent further damage, allowing some hair cell regeneration and recovery of vestibular function. ${ }^{33}$

Our report of 103 cases seen over 23 years suggests that vestibulotoxicity is rare (Addendum). However, our criteria excluded patients in whom we were unable to obtain dosing and clinical details, those with pre-existing renal failure, and those with partial bilateral ${ }^{9}$ or unilateral vestibulotoxicity. ${ }^{34}$

GVT can occur with any dose, in any regimen, at any serum level, and is often not recognised. Vestibulotoxicity can be devastating and conscious patients should be warned of this risk before being treated with gentamicin.

Acknowledgements: We thank Dr Richard Benn for helpful discussions over many years and Dr Vicki Levidiotis for reviewing the manuscript. The study was supported by Garnett Passe and Rodney Williams Memorial Foundation (Hamish MacDougall) and National Health and Medical Research Council Grant 245515 (Michael Halmagyi).

Competing interests: Michael Halmagyi and Hamish MacDougall have acted as unpaid consultants for GN Otometrics.

\section{Received 5 Jul 2011, accepted 5 Mar 2012.}

1 Dobie RA, Black FO, Pezsnecker SC, Stallings VL. Hearing loss in patients with vestibulotoxic reactions to gentamicin therapy. Arch Otolaryngol Head Neck Surg 2006; 132: 253-257.

2 Seemungal BM, Bronstein AM. Aminoglycoside ototoxicity: vestibular function is also vulnerable. BMJ 2007: 335: 952.

3 Halmagyi GM, Fattore CM, Curthoys IS, Wade S. Gentamicin vestibulotoxicity. Otolaryngol Head Neck Surg 1994; 111: 571-574.

4 Black FO, Pesznecker S, Stallings V. Permanent gentamicin vestibulotoxicity. Otol Neurotol 2004 25: 559-569.

5 Ishiyama G, Ishiyama A, Kerber K, Baloh RW. Gentamicin ototoxicity: clinical features and the effect on the human vestibulo-ocular reflex. Acta Otolaryngol 2006; 126: 1057-1061.

6 Ariano RE, Zelenitsky SA, Kassum D. Aminoglycoside-induced vestibular injury: maintaining a sense of balance. Ann Pharmacother 2008; 42: 1282-1289.

7 Minor LB. Gentamicin-induced bilateral vestibular hypofunction. JAMA 1998; 279: 541-544.

8 Zingler VC, Cnyrim C, Jahn K, et al. Causative factors and epidemiology of bilateral vestibulopathy in 255 patients. Ann Neurol 2007: 61: 524-532.

9 Weber KP, Aw ST, Todd MJ, et al. Horizontal head impulse test detects gentamicin vestibulotoxicity. Neurology 2009; 72: 1417-1424.

10 Vital D, Hegemann SC, Straumann D, et al. A new dynamic visual acuity test to assess peripheral vestibular function. Arch Otolaryngol Head Neck Surg 2010; 136: 686-691.

II Vereeck L, Truijen S, Wuyts FL, Van de Heyning PH. The dizziness handicap inventory and its

7 Lateral, anterior and superior semicircular canal vestibulo-ocular reflexes measured with videooculography* during a head impulse test

A. Normal subject
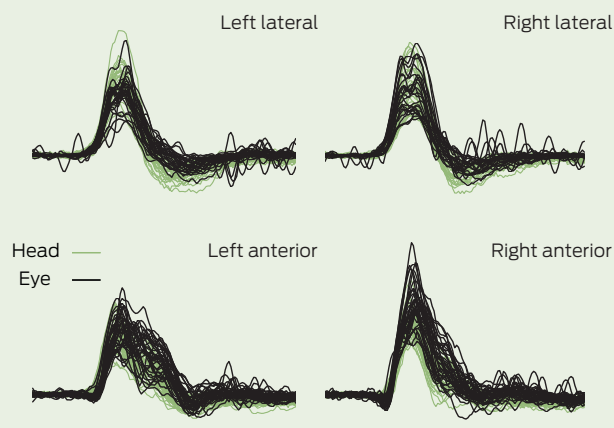

$100 \%$ Left posterior

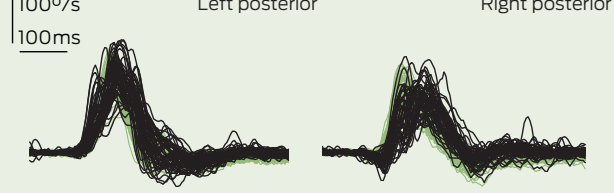

B. Bilateral vestibulotoxicity
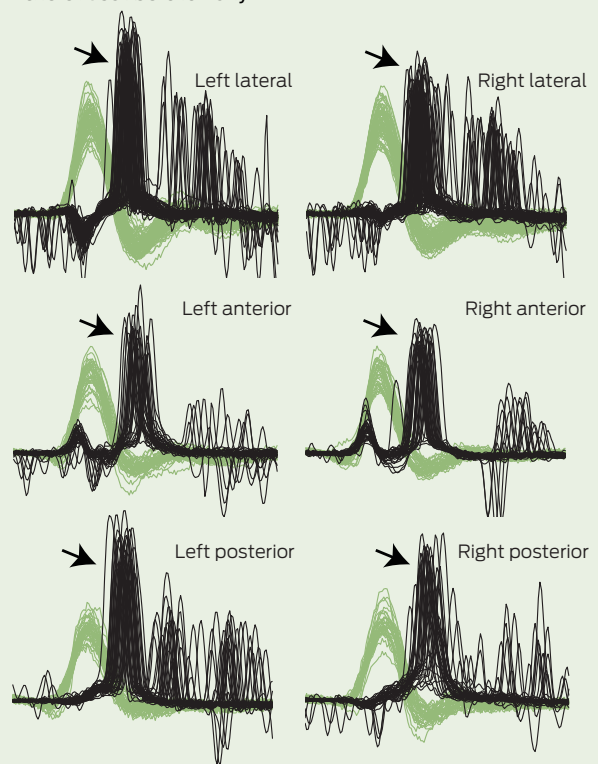

(A) In a normal subject, eye velocity (polarity inverted for ease of comparison) exactly matches head velocity for lateral, anterior and posterior vestibulo-ocular reflexes. (B) In a patient with severe gentamicin vestibulotoxicity, vestibulo-ocular reflexes from all six semicircular canals are severely deficient; patient makes salvos of catch-up saccades (arrows), often visible to the clinician. ${ }^{22}$ *ICS Video vestibulometry, GN Otometrics, Schaumburg, Illinois, USA, and Tåstrup, Denmark.

relationship with functional balance performance. Otol Neurotol 2007; 28: 87-93.

12 Halmagyi GM, Yavor RA, McGarvie LA. Testing the vestibulo-ocular reflex. Adv Otorhinolaryngol 1997; 53: 132-154.

13 Antibiotic Expert Group. Therapeutic guidelines: antibiotic. Version 14. Melbourne: Therapeutic Guidelines Limited, 2010.

14 Herdman SJ. Vestibular rehabilitation. 3rd ed. New York: Davis, 2007: 309-359.

15 Gates, GA, Cooper JC, Kannel WB, Miller NJ. Hearing in the elderly: the Framingham cohort, 1983-1985. Part I. Basic audiometric test results. Ear Hear 1990; 11: 247-256.

16 Pearson JD, Morrell CH, Gordon-Salant S, et al. Gender differences in a longitudinal study of ageassociated hearing loss. J Acoust Soc Am 1995; 97 : 1196-1205.

17 Sha SH, Qiu JH, Schacht J. Aspirin to prevent gentamicin-induced hearing loss. N Engl J Med 2006; 354: 1856-1857.

18 Moore RD, Smith CR, Lietman PS. Risk factors for the development of auditory toxicity in patients receiving aminoglycosides.J Infect Dis 1984; 149: 23-30.

19 Lancaster JL, Mortimore S, McCormick M, Hart CA. Systemic absorption of gentamicin in the management of active mucosal chronic otitis media. Clin Otolaryngol Allied Sci 1999; 24 : 435-439.

20 Stavroulaki P, Apostolopoulos N, Dinopoulou D, et al. Otoacoustic emissions - an approach for monitoring aminoglycoside induced ototoxicity in children. Int J Pediatr Otorhinolaryngol 1999; 50: 177-184.

21 JC. Living without a balancing mechanism. NEngl JMed 1952; 246: 458-460.

22 MacDougall HG, Weber KP, McGarvie LA, et al. The video head impulse test: diagnostic accuracy in peripheral vestibulopathy. Neurology 2009; 73 1134-1141.

23 Gentamicin Information Center. About gentamicin poisoning. http://www.gentamicin. com (accessed Mar 2012).

\section{Addendum (after acceptance)}

We have seen two further patients with severe bilateral gentamicin vestibulotoxicity.

An 87-year-old woman ( $57 \mathrm{~kg}$ and eGFR $>60 \mathrm{~mL} / \mathrm{min} / 1.73 \mathrm{~m}^{2}$ ) was given gentamicin prophylaxis before $(240 \mathrm{mg})$ and after $(160 \mathrm{mg})$ aortic valve replacement surgery in late 2009. Caloric responses are now absent. Moderately severe, bilateral, symmetrical sensorineural hearing loss is unchanged from an audiogram in 2005. She is now severely disabled by imbalance and virtually housebound.

A 60-year-old woman ( $60 \mathrm{~kg}$ and eGFR, > $60 \mathrm{~mL} / \mathrm{min} / 1.73 \mathrm{~m}^{2}$ ) was given gentamicin ( $400 \mathrm{mg}$ one day and $320 \mathrm{mg}$ the next day) for febrile neutropenia after chemotherapy for breast cancer in early 2012. Her caloric and rotational responses are absent; her hearing is consistent with age. She is moderately disabled, but able to participate in home-based vestibular rehabilitation exercises.

24 Appel GB. Aminoglycoside nephrotoxicity. Am J Med 1990; 88: 16S-20S

25 Prins JM, Buller HR, Kuijper EJ, et al. Once versus thrice daily gentamicin in patients with serious infections. Lancet 1993; 341: 335-339.

26 Raveh D, Koypt M, Hite Y, et al. Risk factors for nephrotoxicity in elderly patients receiving once-daily aminoglycosides. QJM 2002; 95: 291-297.

27 English WP, Williams MD. Should aminoglycoside antibiotics be abandoned? Am J Surg. 2000; 180: 512-515

28 Leong CL, Buising K, Richards M, et al. Providing guidelines and education is not enough: an audit of gentamicin use at The Royal Melbourne Hospital. Intern Med J 2006; 36: 37-42

29 Dunagan WC, Woodward RS, Medoff G, et al. Antibiotic misuse in two clinical situations: positive blood culture and administration of aminoglycosides. Rev Infect Dis 1991; 13: 405-412.

30 Johnson MW, Mitch WE, Heller AH, Spector R. The impact of an educational program on gentamicin use in a teaching hospital. Am J Med 1982; 73: 9-14

31 Wu WJ, Sha SH, Schacht J. Recent advances in understanding aminoglycoside ototoxicity and its prevention. Audiol Neurootol 2002; 7: 171-174

32 Chen FQ, Schacht J, Sha SH. Aminoglycoside-induced histone deacetylation and hair cell death in the mouse cochlea. J Neurochem 2009; 108: 1226-1236.

33 Black FO, Gianna-Poulin C, Pesznecker SC. Recovery from vestibular ototoxicity. Otol Neurotol 2001; 22: 662-671.

34 Ahmed RM, MacDougall HG, Halmagyi GM. Unilateral gentamicin vestibulotoxicity. Otol Neurotol 2011; 32: 1158-1162. 
Research

1 Definitions of severe bilateral vestibular loss

Caloric testing: < 4\% peak nystagmus slow-phase velocity from each ear in response to $30^{\circ} \mathrm{C}$ and $44^{\circ} \mathrm{C}$ stimulation or $<6 \%$ in response to $0^{\circ} \mathrm{C}$ stimulation, or both.

Rotational testing: < 10\%s peak nystagmus slow-phase velocity, or time constant of $<8 \mathrm{~s}$, or both, in response to a constant acceleration stimulus of $20 \% \mathrm{~s}^{2}$ for $5 \mathrm{~s}$, or $40 \% / \mathrm{s}^{2}$ for $3 \mathrm{~s}$ or $100 \% / \mathrm{s}^{2}$ for $1 \mathrm{~s}$.

2 Hearing by age in 73 patients with gentamicin vestibulotoxicity (two ears) - average and range of lowest pure-tone thresholds in decibels at 500-8000 Hertz

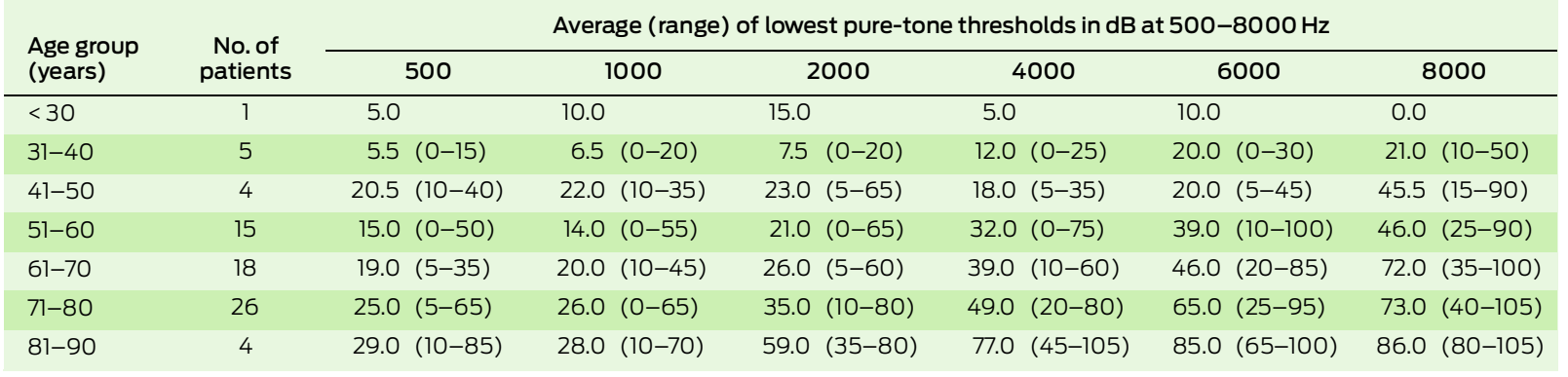

3 Total dose $(\mathrm{mg} / \mathrm{kg})$ versus daily dose $(\mathrm{mg} / \mathrm{kg} /$ day) of gentamicin in 103 patients with vestibulotoxicity

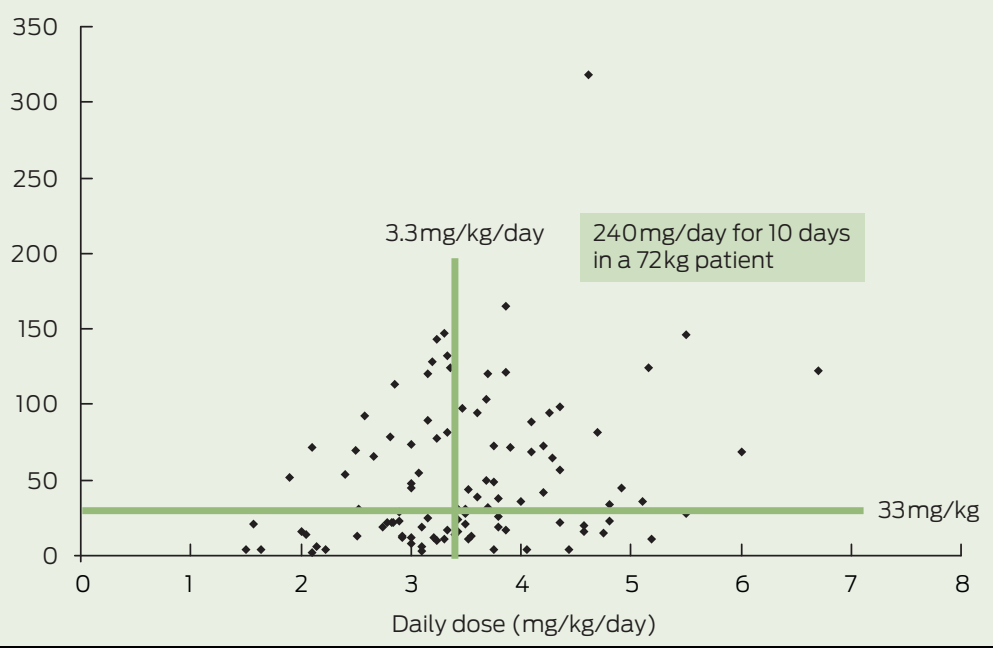

The horizontal and the vertical lines showing total dose $(33 \mathrm{mg} / \mathrm{kg})$ and daily dose $(3.3 \mathrm{mg} / \mathrm{kg} / \mathrm{day})$, respectively, represent a typical dose of $240 \mathrm{mg} /$ day given for 10 days to a $72 \mathrm{~kg}$ patient. Note that 50 patients had less than this total dose, 48 patients had less than this daily dose, and 29 patients had both. 
- Research

706 MJA $196(11) \cdot 18$ June 2012 